\title{
EfficAcy and Safety of Tripterygium Wilfordii Multiglycosides in Idiopathic Membranous Nephropathy (EAST): Protocol for An Open-Label Randomized Controlled Clinical Trial
}

\section{Chanyu Geng}

Sichuan Provincial People's Hospital: Sichuan Academy of Medical Sciences and Sichuan People's Hospital https://orcid.org/0000-0002-4892-0944

Yunlin Feng ( $\square$ fengyunlin@med.uestc.edu.cn )

Sichuan Provincial People's Hospital: Sichuan Academy of Medical Sciences and Sichuan People's Hospital

Qiang Li

The George Institute for Global Health

Lei Pu

Sichuan Provincial People's Hospital: Sichuan Academy of Medical Sciences and Sichuan People's Hospital

Hongling Yang

Sichuan Provincial People's Hospital: Sichuan Academy of Medical Sciences and Sichuan People's Hospital

\section{Guisen Li}

Sichuan Provincial People's Hospital: Sichuan Academy of Medical Sciences and Sichuan People's Hospital

\section{Research Article}

Keywords: tripterygium wilfordii multiglycosides, idiopathic membranous nephropathy, open-label, randomized controlled trial, efficacy, safety

Posted Date: October 25th, 2021

DOI: https://doi.org/10.21203/rs.3.rs-889263/v1

License: (c) (1) This work is licensed under a Creative Commons Attribution 4.0 International License. Read Full License

Version of Record: A version of this preprint was published at Medicine on February 11th, 2022. See the published version at https://doi.org/10.1097/MD.0000000000028842. 


\section{Abstract \\ Background}

Idiopathic membranous nephropathy (IMN) is one of the most common primary glomerular diseases in China, especially in the elderly. Patients who are classified as low risk of renal function decline based on KDIGO guidelines often present with obvious lower extremities edema, which make the supportive treatment of RAS inhibitors less accepted. Tripterygium wilfordii multiglycosides has been used to treat rheumatic disease over years in China, and has been demonstrated to have the effect of reducing proteinuria and alleviate edema in patients with chronic kidney disease.

\section{Methods}

This is an open-labeled, randomized, controlled clinical trial. Twenty patients with IMN who meet the inclusive criteria will be randomly assigned into the intervention group and the control group at a rate of 1:1. The intervention group was treated with tripterygium wilfordii multiglycosides tablets $(1-1.5 \mathrm{mg} / \mathrm{kg}$ body weight, oral administration after three meals) in addition to the original dose of ACEi/ARB, while the control group continued with the original dose of ACEi/ARB. The treatment course is six months, and the clinical data of patients will be collected at baseline and at each monthly follow-up. The primary outcome measure is the absolute value of relative baseline decrease of urinary protein quantity after six months treatment. The secondary outcome measures include absolute value of relative baseline decrease of ACR in morning urine after 6 months treatment, the percentage of patients who reached the standard of effective clinical treatment, and complex renal end point events. Safety outcome measures include the incidence of adverse events, the incidence of serious adverse events, changes in laboratory safety indicators, and death.

\section{Discussion}

This study aims to provide higher-level evidence of evidence-based medicine for tripterygium wilfordii multiglycosides in the treatment of this disease, and hopefully has important significance for clinicians who are to make therapeutic decisions of IMN.

\section{Trial Registration:}

This trial has been prospectively registered in Chinese Clinical Trial Registry (identifier\# ChiCTR2100048382) on 6 July 2021.

\section{Trial status:}

Recruiting. Recruitment is expected to be completed in June 2020.

\section{Background}

Idiopathic membranous nephropathy (IMN) is one of the common pathological types of primary nephrotic syndrome in adults, with a peak age of onset around $40-50$ years old [1-2]. In China, IMN accounts for $30.2 \%$ of primary glomerular disease and is the leading cause of nephrotic syndrome in adults over 40 years old[3]. Immunosuppressive therapy is now the first-line treatment of IMN, and its efficacy has been confirmed by a large number of high-level evidences[4]. The latest version of KDIGO guideline of Chronic Glomerular Disease [5] recommends that immunosuppressive therapy should be reserved for IMN patients at moderate to high risk for progressive renal function decline, but not for initiation therapy for IMN patients with proteinuria < $3.5 \mathrm{~g} / \mathrm{d}$ and eGFR $>60 \mathrm{ml} / \mathrm{min} / 1.73 \mathrm{~m}^{2}$. However, we have found a considerable number of patients, whose proteinuria were less than 3.5 grams per day, suffered from significant edema, making the acceptance for supporting treatment of full-dose RAS inhibitors including angiotensin converting enzymes inhibitors (ACEi) or angiotensin receptor blockers (ARB) relatively low in this population, especially in the elderly. 
Tripterygium wilfordii multiglycosides is extracted and purified from the root xylem of tripterygium wilfordii Hook F (TwHF), which is a member of the Celastraceae family of perennial vine-like plants[6]. We have observed in clinical practise and it has also been reported in literature that tripterygium wilfordii multiglycosides can reduce urine protein and alleviate edema of patients with chronic kidney disease[7-9], through mechanisms including but not limited to activating $T$ cells [10] and regulating NF-K B pathway [7, 10-11]. Most experience of tripterygium wilfordii multiglycosides in IMN so far came from observational studies with limited number of subjects. There is still a lack of solid evidence to support its use in IMN patients.

Therefore, we designed this open-label randomized controlled trial to investigate the efficacy and safety of tripterygium wilfordii multiglycosides in IMN patients who would be classified as low-risk according to KDIGO guideline, aiming to deepen the understanding of this drug in treating IMN and provide evidence for relevant practice.

\section{Methods}

The protocol was developed following superiority trial design, to compare the efficacy and safety of tripterygium wilfordii multiglycosides over RAS inhibitors in IMN.

\section{Study setting}

The EAST -trial will be performed in Sichuan Provincial People's Hospital. Patients are recruited at the nephrology clinic if they meet the criteria as defined below.

\section{Design}

This is an open-label randomized controlled clinical trial in a single tertiary center. 20 eligible patients with IMN will be randomly assigned to an intervention group and a control group at a rate of 1:1, with ten patients in each group. The intervention group will be receive tripterygium wilfordii multiglycosides tablets on the basis of RAS inhibitors, while the control group will continue with RAS inhibitors. The study duration will be six months, during which clinical data and samples will be collected according to a visit schedule at each follow-up. A flowchart of the trial design is shown in Figure 1.

\section{Inclusive criteria}

Patients diagnosed as IMN by renal biopsy will be included in this study. Inclusive criteria are: (1) has no evidence of secondary membranous nephropathy; (2) Age between 18 to 65 years old; (3) has 24-hour albuminuria between 1.0 and $6.0 \mathrm{~g}$ after at least three month of standard dose of RAS inhibitor treatment; (4) estimated glomerular filtration rate (eGFR) $>60 \mathrm{ml} / \mathrm{min} / 1.73 \mathrm{~m}{ }^{2}$ according to CKD-EPI formula using the latest serum creatinine value at screening $\mathbb{( 5 )}$ has not participated in other clinical trials within the last three months; (5) has negative pregnancy test result for women of childbearing age; and (6) agrees to voluntarily participate in the study and signs an informed consent form.

\section{Exclusive criteria}

Patients with any one of the following criteria will be excluded: (1) has received glucocorticoids or other immunosuppressants in the past six months; (2) has any clinical evidence of secondary membranous nephropathy (tumors, drugs, other autoimmune diseases such as systemic lupus erythematosus, etc.); (3) has any of the following comorbidities including chronic liver disease, myocardial infarction, stroke, uncontrolled hypertension and diabetes, malignant tumor; (4) is known to allergic to tripterygium wilfordii multiglycosides or has contraindications for use; (5) female patients who are pregnant (or intend to be pregnant within half a year), breastfeeding or unwilling to take contraceptive measures; (6) has an expected life expectancy less than six months; (7) is currently participating or have participated in other clinical trials within 3 months; (8) refuses to sign an informed consent form or be unable or unwilling to comply with research protocol approved by the researcher; (9) has any other conditions which the researchers believe makes the subject unsuitable for this study.

\section{Sample size}


We identified a total of 87 IMN patients by screening renal biopsy data and clinical follow-up data from January 2010 to December 2017 in our center's Renal Treatment System. The average amount of proteinuria per day in this population was 2.89 $\pm 0.62 \mathrm{~g}$ at the time of diagnosis. According to the clinical data of our center, combined with the published data on proteinuria in patients with IMN, we expect the mean decrease of proteinuria in the intervention group being $2.0 \mathrm{~g} /$ day higher than that in the control group to be considered as effective. Therefore, we set a standard deviation (s) of $1.5 \mathrm{~g} /$ day, the necessary sample size in each group to provide a power of $80 \%$ to detect an effect size of $2 \mathrm{~g} /$ day, with type I error $(\mathrm{a})=0.05$ (bilateral) and 1:1 randomization was calculated as follows:

$$
\mathrm{n}=2\left[\frac{(\mu 1-\alpha+\mu 1-\beta) s}{\varepsilon}\right] \wedge 2=2\left[\frac{(1.96+0.84) \times 1.5}{2}\right] \wedge 2=8.8 \approx 9
$$

The total number increased to 20 cases considering a loss of follow-up rate of $10 \%$. Therefore, 20 subjects will be included in this study.

\section{Randomization}

A random number list will be generated by SPSS version 24.0 (IBM Corporation, USA) using simple randomization. Eligible patients will be randomly assigned to the interventional group or the control group at a rate of 1:1 by the number inside a concealed envelope containing random codes according to the order of enrollment.

\section{Interventions}

All patients will have received ACEi/ARB treatment for at least three months before enrollment. After randomization, patients in the interventional group will receive tripterygium wilfordii multiglycosides tablets $(1-1.5 \mathrm{mg} / \mathrm{kg}$ body weight/day, per oral, divided to three time daily) in addition to their ACEi/ARB treatment, and patients in the control group will proceed with their ACEi/ARB treatment. The overall treatment will last for six months.

\section{Withdraw criteria}

Patients with any of the following conditions will be withdrawn from this study: (1) being found having any of the exclusive criteria after enrollment; (2) takes glucorsteroids or other immunosuppressants during the study; (3) has intolerable side effects of drugs; (4) has any serious adverse event that makes subjects unsuitable for continuation, such as severe infection, uncontrollable hyperglycemia and/or hypertension; (5) the patient asks to withdraw; (6) other situations which the researchers believe makes the patient not appropriate to continue.

\section{Efficacy outcome measures}

The primary outcome measure for efficacy is absolute decrease of proteinuria after six months.

The secondary outcome measures include:

- absolute decrease of urine albumin-to-creatinine ratio (ACR) after six months;

- the percentage of patients who have achieved complete response, defined as proteinuria less than $0.3 \mathrm{~g} / \mathrm{day}$ and serum albumin $\geq 3.5 \mathrm{~g} / \mathrm{dL}$;

- the percentage of patients who have achieved partial response, defined as a decrease of proteinuria $\geq 50 \%$ compared with baseline level and proteinuria between 0.3 to $3.5 \mathrm{~g} /$ day.

\section{Safety outcomes measures}

Since the main adverse effects of tripterygium wilfordii multiglycosides is liver dysfunction and gonadal suppression [12,13], we will closely monitor manifestations of these two aspects in addition to routine practice on safety surveillance. Therefore, 
safety parameters in this study will include but not limited to the following:

- Clinical: blood pressure, edema, anemia, menstruation status in the females;

- Blood biological tests: CBC, creatine, albumin, alanine aminotransferase, aspartic aminotransferase, total bilirubin, direct bilirubin, indirect bilirubin, lactate dehydrogenase;

- Urine biological tests: red blood cells, white blood cells.

\section{Assessment}

All enrolled patients will have their first visit at the time of screening, the second visit at randomization and be followed up at the end of each month afterwards. In addition, there will be one follow up at two weeks after randomization (Visit 3), of which the main purpose is to closely monitor potential adverse effect of tripterygium wilfordii multiglycosides. The overall duration of treatment will be six months. At each follow-up as shown in Table 1, the medical history will be enquired, and blood and urine tests will be carried out in the central lab in Sichuan Provincial People's Hospital with standardized methods.

Table 1 Visit arrangement.

Research program Screening Randomization Clinic Visit

$\begin{array}{lllllllll}1 & 2 & 3 & 4 & 5 & 6 & 7 & 8 & 9\end{array}$

Visit number

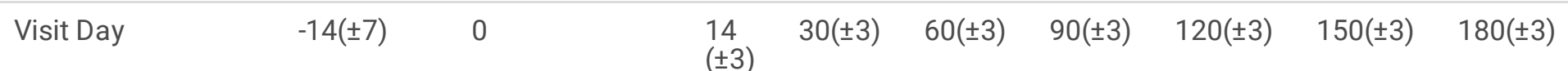

\begin{tabular}{lll} 
Informed consent & $\sqrt{ }$ & \\
$\begin{array}{l}\text { Inclusion/Exclusive } \\
\text { criteria }\end{array}$ & $\sqrt{ }$ & $\sqrt{ }$ \\
$\begin{array}{l}\text { Random } \\
\text { assignment }\end{array}$ & \\
\hline
\end{tabular}

\begin{tabular}{|c|c|c|c|c|c|c|c|c|c|}
\hline Demographics & $\sqrt{ }$ & & & & & & & & \\
\hline Medical history & $\sqrt{ }$ & $\sqrt{ }$ & $\sqrt{ }$ & $\sqrt{ }$ & $\sqrt{ }$ & $\sqrt{ }$ & $\sqrt{ }$ & $\sqrt{ }$ & $\sqrt{ }$ \\
\hline $\begin{array}{l}\text { Physical } \\
\text { examination }\end{array}$ & $\sqrt{ }$ & $\sqrt{ }$ & $\sqrt{ }$ & $\sqrt{ }$ & $\sqrt{ }$ & $\sqrt{ }$ & $\sqrt{ }$ & $\sqrt{ }$ & $\sqrt{ }$ \\
\hline Vital signs & $\sqrt{ }$ & $\sqrt{ }$ & $\sqrt{ }$ & $\sqrt{ }$ & $\sqrt{ }$ & $\sqrt{ }$ & $\sqrt{ }$ & $\sqrt{ }$ & $\sqrt{ }$ \\
\hline Blood lab tests* & & $\sqrt{ }$ & $\sqrt{ }$ & $\sqrt{ }$ & $\sqrt{ }$ & $\sqrt{ }$ & $\sqrt{ }$ & $\sqrt{ }$ & $\sqrt{ }$ \\
\hline Urine lab tests* & & $\sqrt{ }$ & $\sqrt{ }$ & $\sqrt{ }$ & $\sqrt{ }$ & $\sqrt{ }$ & $\sqrt{ }$ & $\sqrt{ }$ & $\sqrt{ }$ \\
\hline $\begin{array}{l}\text { Collect blood and } \\
\text { urine samples }\end{array}$ & & $\sqrt{ }$ & & & $\sqrt{ }$ & & $\sqrt{ }$ & & $\sqrt{ }$ \\
\hline Pregnancy test & $\sqrt{ }$ & $\sqrt{ }$ & & & & & & & \\
\hline $\begin{array}{l}\text { Combined } \\
\text { medication }\end{array}$ & & $\sqrt{ }$ & $\sqrt{ }$ & $\sqrt{ }$ & $\sqrt{ }$ & $\sqrt{ }$ & $\sqrt{ }$ & $\sqrt{ }$ & $\sqrt{ }$ \\
\hline Adverse events & & $\sqrt{ }$ & $\sqrt{ }$ & $\sqrt{ }$ & $\sqrt{ }$ & $\sqrt{ }$ & $\sqrt{ }$ & $\sqrt{ }$ & $\sqrt{ }$ \\
\hline Drug distribution & & $\sqrt{ }$ & $\sqrt{ }$ & & $\sqrt{ }$ & $\sqrt{ }$ & $\sqrt{ }$ & $\sqrt{ }$ & \\
\hline $\begin{array}{l}\text { Drug compliance } \\
\text { reminder }\end{array}$ & & $\sqrt{ }$ & $\sqrt{ }$ & & $\sqrt{ }$ & $\sqrt{ }$ & $\sqrt{ }$ & $\sqrt{ }$ & \\
\hline
\end{tabular}




\section{Data management}

In this study, the paper version of CRF table will be used for data collection, RedCap online database will be used to manage data, double input comparison method will be used for data entry and verification, and corresponding data verification and cleaning will be carried out. During the study period, the principal researchers will check the trial data once a month to ensure internal consistency.

\section{Data analysis}

Continuous data will be described by mean \pm standard deviation or median (interquartile range). Classified data will be described by the number and percentages. Chi-square test (Fisher's Exact test, if the expected number $<5$ ) for categorical variables and Student's t-test or Wilcoxon Rank Sum test for continuous variables will be applied to detect the differences between the intervention and the control groups. A two-tailed $p<0.05$ will be considered to be significant. Statistical analysis will be performed using SPSS version 24.0 (IBM Corporation, Armonk, NY) and RStudio for Windows, version 4.0.3 (RStudio, Inc.) and supervised by a skilled statistician.

\section{Ethnic Consideration}

The study received approval from the Ethics Committee of Sichuan Provincial people's Hospital (No. 2021.325-1). The study will be conducted following Ethical Principles of Helsinki Declaration and relevant local clinical research norms and regulations. Written informed consent will be acquired from every participant before study-related procedure. A verification of the consents will be carried out after each inclusion, followed by an auditing of the files at regular time intervals. The participants' personal data will be kept confidential throughout the study and only available to the research team. Any change to the protocol should be approved by the Ethics Committee before it can be implemented and will be applied to the clinical registry for protocol changes.

\section{Discussion}

IMN is one of the most common primary glomerular diseases in China, especially in the elderly, and its prevalence is rising over recent years $[14,15]$. Patients who are classified as low risk of renal function decline based on KDIGO guidelines often present with obvious lower extremities edema, which make the supportive treatment of RAS inhibitors less accepted.

Tripterygium wilfordii multiglycosides has been used to treat rheumatic disease over years. Evidence from a number of observational studies indicated this drug can reduce urine protein and alleviate edema of patients with chronic kidney disease including IMN [12], the fact with which our own observations consist. Previous studies have shown that tripterygium wilfordii multiglycosides can significantly reduce the production of inflammatory cytokines such as TNF- $\alpha$, IL-1 $\beta$ and MCP-1 and ameliorated oxidative stress[10]. It can attenuate inflammatory response by down-regulation of NF-KB signaling pathway[10, 11]. In addition, tripterygium wilfordii multiglycosides can also promote the structural repair of podocyte hiatus membrane protein [7]. These mechanisms might explain the reduction of proteinuria observed. However, there is still a lack of high-level evidence for using this drug to treat IMN. Therefore, we designed this open-label, randomized, controlled trial to investigate its efficacy and safety in treating this population.

Tripterygium wilfordii multiglycosides has known side effects, of which hepatotoxicity and menstrual disorder are the top two concerned. Excessive dose and cumulative dose are the main causes of adverse effects [13]. The highest incidence of reproductive toxicity among tripterygium wilfordii multiglycosides users thus far reported was 7.9\% [14]. In our study, we will strictly calculate and control the dose and treatment duration of each subject to avoid drug overdose and accumulation. Meanwhile, we will closely monitor adverse effects in monthly follow up visit, and we also add one follow-up (Visit 3) to closely monitor potential adverse effects of tripterygium wilfordii multiglycosides. If intolerable side effects occurred, we will withdraw the subject immediately, take appropriate measures and follow up until the side effect resolves. 
It should be noted the calculated sample size is relatively small, only ten for each group considering a loss of follow-up rate of $10 \%$. Thus, strict adherence to study protocol and every effort to keep the patient followed up as planned are of great importance to the success of this trial.

In summary, This study aims to provide scientific evidence for tripterygium wilfordii multiglycosides in treating IMN through an open-labeled, randomized, controlled clinical trial. The results may provide higher-level evidence of evidence-based medicine for tripterygium wilfordii multiglycosides in the treatment of this disease, and hopefully have important significance for clinicians who are to make therapeutic decisions of IMN.

\section{Trial Status}

Trial status EAST version 1 on 22 August 2021. The study is now recruiting. Recruitment is expected to be completed in June 2022.

\section{List of abbreviations}

IMN: Idiopathic membranous nephropathy

TwHF: tripterygium wilfordii Hook F

ACEi: angiotensin converting enzymes inhibitors

ARB: angiotensin receptor blockers

TW: tripterygium wilfordii multiglycosides

ACR: albumin-to-creatinine ratio

\section{List Of Abbreviations}

IMN: Idiopathic membranous nephropathy

TwHF: tripterygium wilfordii Hook F

ACEi: angiotensin converting enzymes inhibitors

ARB: angiotensin receptor blockers

TW: tripterygium wilfordii multiglycosides

ACR: albumin-to-creatinine ratio

\section{Declarations}

\section{Authors' contributions}

YLF conceived the study and wrote the manuscript. CYG wrote the manuscript and led the registration. QL, LP, HLY and GSL contributed to the study design and critically reviewed the manuscript. All authors read and approved the final manuscript.

\section{Funding}

The study is financially supported by the PRO-Run Foundation of Nephrology Group of Evidence-based Medicine Committee of Chinese Physicians Association (Grant No. KYS2021-03-02-2). The sponsor has no role in the design and conduct of the study; 
in the collection, management, analysis, and interpretation of the data; or in the preparation, review, or approval of the manuscript.

\section{Availability of data and materials}

All authors will have access to the final trial dataset.

\section{Ethics approval and consent to participate}

The study received approval from the Ethics Committee of Sichuan Provincial people's Hospital (No. 2021.325-1). The study will be conducted following Ethical Principles of Helsinki Declaration and relevant local clinical research norms and regulations. Written informed consent will be acquired from every participant before study-related procedure. A verification of the consents will be carried out after each inclusion, followed by an auditing of the files at regular time intervals. The participants' personal data will be kept confidential throughout the study and only available to the research team. Any change to the protocol should be approved by the Ethics Committee before it can be implemented and will be applied to the clinical registry for protocol changes.

\section{Consent for publication}

Not applicable

\section{Competing interests}

All authors declare they do not have any other financial and personal conflicts of interest with this manuscript.

\section{Acknowledgements}

Not applicable.

\section{References}

1. Glassock RJ. Diagnosis and natural course of membranous nephropathy. Semin Nephrol. 2003;23(4):324-32. doi:10.1016/s0270-9295(03)00049-4.

2. Lai WL, Yeh TH, Chen PM, et al. Membranous nephropathy: a review on the pathogenesis, diagnosis, and treatment. J Formos Med Assoc. 2015;114(2):102-11. doi:10.1016/j.jfma.2014.11.002.

3. Xu X, Wang G, Chen N, et al. Long-Term Exposure to Air Pollution and Increased Risk of Membranous Nephropathy in China. J Am Soc Nephrol. 2016;27(12):3739-46. doi:10.1681/ASN.2016010093.

4. Zheng Q, Yang H, Liu W, et al. Comparative efficacy of 13 immunosuppressive agents for idiopathic membranous nephropathy in adults with nephrotic syndrome: a systematic review and network meta-analysis. BMJ Open. 2019;9(9):e030919. doi:10.1136/bmjopen-2019-030919. Published 2019 Sep 11.

5. KDIGO Clinical Practice Guideline On. Glomerular Diseases Public Review Draft June 2020.

6. Song $C Y, X u$ YG, Lu YQ. Use of Tripterygium wilfordii Hook F for immune-mediated inflammatory diseases: progress and future prospects. J Zhejiang Univ Sci B. 2020;21(4):280-90. doi:10.1631/jzus.B1900607.

7. Guo XY, Sun GY, Cai YR, Wang RK. Experimental Research Progress on the Mechanism of traditional Chinese Medicine in the treatment of Idiopathic membranous Nephropathy based on podocyte Protection. Chinese Journal of Integrated Traditional and Western Nephrology. 2017 (04):374-376. (in Chinese).

8. Liu S, Li X, Li H, Liang Q, Chen J, Chen J. Comparison of tripterygium wilfordii multiglycosides and tacrolimus in the treatment of idiopathic membranous nephropathy: a prospective cohort study. BMC Nephrol. 2015;16:200. doi:10.1186/s12882-015-0199-x. Published 2015 Dec 4.

9. Shang SL, Cai GY, Duan SW, Li P, Li QG, Chen XM. Retrospective analysis of tacrolimus combined with Tripterygium wilfordii polyglycoside for treating idiopathic membranous nephropathy. BMC Nephrol. 2018;19(1):182. Published 2018 Jul 
18. doi:10.1186/s12882-018-0967-5.

10. Zhou Y, Hong Y, Huang H. Triptolide Attenuates Inflammatory Response in Membranous Glomerulo-Nephritis Rat via Downregulation of NF-kB Signaling Pathway. Kidney Blood Press Res. 2016;41(6):901-10. doi:10.1159/000452591.

11. Wan Q, Liu Z, Yang M, Deng P, Tang N, Liu Y. Triptolide ameliorates fine particulate matter-induced podocytes injury via regulating NF-KB signaling pathway. BMC Mol Cell Biol. 2020 Feb 3;21(1):4. doi: 10.1186/s12860-020-0248-6. PMID: 32013860; PMCID: PMC6998203.

12. Wang D, Zhao XH, Cui Y, Zhang TT, Wang F, Hu YH. Efficacy and safety of Tripterygium wilfordii Hook F for CKD in Mainland China: A systematic review and meta-analysis. Phytother Res. 2018;32(3):436-51. doi:10.1002/ptr.5987.

13. Tian YG, Su XH, Liu LL, Kong XY, Lin N. Zhongguo Zhong Yao Za Zhi. 2019;44(16):3399-405. doi:10.19540/j.cnki.cjcmm.20190527.408.

14. Xu Y, Fan YF, Zhao Y, Lin N. Zhongguo. Zhong Yao Za Zhi. 2019;44(16):3406-14. doi:10.19540/j.cnki.cjcmm.20190524.401.

\section{Figures}

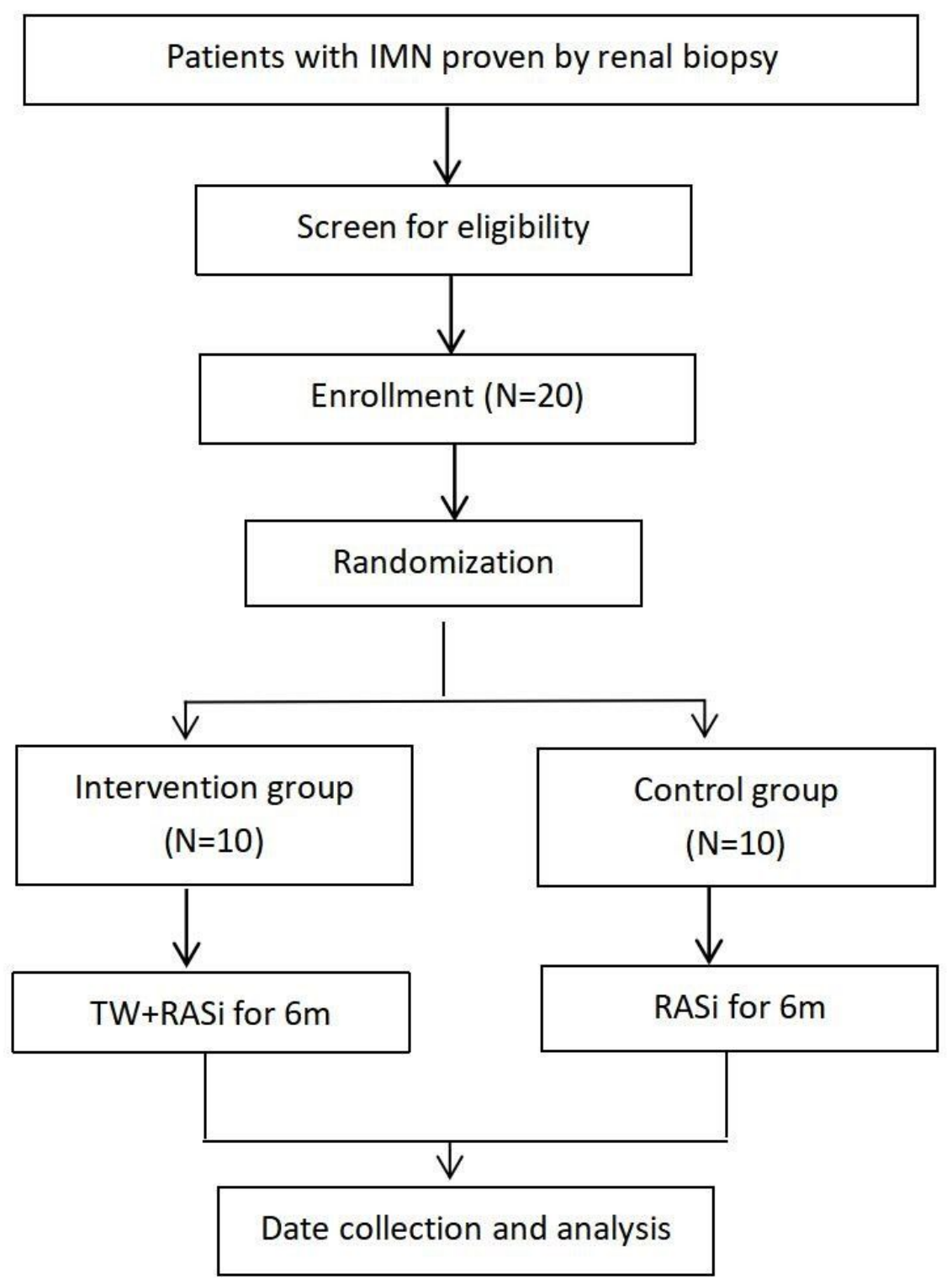




\section{Figure 1}

Flowchart of the trial. Abbreviations: m, months; RASi, renin-angiotensin system inhibitors; TW, tripterygium wilfordii multiglycosides

\section{Supplementary Files}

This is a list of supplementary files associated with this preprint. Click to download.

- SPIRITchecklist.docx 